\title{
Application of computer-aided image analysis to studies of arbuscular endomycorrhizal fungi effects on plant root system morphology and dynamics
}

\author{
JE Hooker, D Atkinson \\ University of Aberdeen, Department of Agriculture, 581 King Street, Aberdeen, AB9 1UD, UK
}

(COST Meeting, 21-23 May 1992, Dijon, France)

\begin{abstract}
Summary - Studies on the effects of arbuscular mycorrhizal (AM) fungi on plant root systems are frequently limited by the availability of techniques. The complex organisation of root systems and their inaccessibility below ground render it technically difficult to make accurate measurements of either root morphology or root dynamics. The 2 systems described both utilise image analysis. One makes possible detailed measurements of root morphology on harvested root samples, permitting quantitative relationships between different hierarchical orders to be determined. The other utilises a miniature colour television camera together with database software to make in situ measurements of root dynamics using minirhizotrons.
\end{abstract}

AM / root morphology / image analysis / root dynamics

Résumé - Application de l'imagerie assistée par ordinateur aux études sur les effets des champignons endomycorhiziens arbusculaires sur la morphologie et la dynaique du système racinaire des plantes. Les études portant sur les effets des mycorhizes arbusculaires sur le système racinaire des plantes sont fréquemment limitées par la validité des techniques disponibles. L'organisation complexe des systèmes racinaires et leur inaccessibilité dans le sol rendent techniquement difficiles les mesures précises, à la fois de la morphologie et de la dynamique des racines. Les 2 systèmes décrits utilisent l'analyse par images. Le premier rend possible les détaillées de la morphologie sur des échantillons de racine prélevés, ce qui permet de déterminer des relations quantitatives entre les différents ordres hiérarchiques. Le second utilise une caméra video miniature en couleurs, associé à un logiciel de base de données pour faire, in situ, des mesures sur la dynamique racinaire en utilisant des minirhizotrons.

mycorhize arbusculaire / morphologie racinaire / analyse d'image / dynamique des racines

\section{INTRODUCTION}

The structure and dynamics of an individual plant's root system has a significant effect on its ability to obtain water and acquire and cycle nutrients. Root diameter and length are important in determining the basic uptake potential of a root system while structural and spatial morphology has a major impact upon actual uptake (Atkinson, 1989). The dynamics of the root system, eg the length of time roots live, the time of year when they grow, are also important; maximum potential uptake will occur when the growth and activity of the root system is synchronized with nutrient and water availability in the soil (Atkinson, 1990).

Recent studies have reported significant increases in the branching of roots as a result of colonisation by AM fungi (Berta et al, 1990; Hooker et al,1992) which do not seem to be en- tirely due to improved host plant nutrition (Hooker et al, 1992). The extent of these changes make it likely that the dynamics of the root system will also be altered. Roots of plants produced by micropropagation are not usually colonised by mycorrhizal fungi. The application of AM inoculum to cultures could therefore produce modifications in root morphology and dynamics which could aid the establishment and growth of plantlets.

Studies on these AM-induced changes are limited by the availability of techniques. Measurements of root morphology are difficult to describe and quantify, as a consequence of the organisational complexity of root systems whilst measurements of root dynamics are difficult to make due to inaccessibility of the below-ground environment (Atkinson and Dawson, 1991).

This paper reports on 2 image-analysis based systems which make it possible to obtain these measurements. 


\section{ROOT MORPHOLOGY}

One of the main difficulties in characterising the structural and quantitative morphology of plant root systems is accurately determining the length of individual root orders. Primarily this is due to the lengths involved, the relatively small diameter of many of the roots and the inevitability of roots crossing over each other and thus concealing other roots underneath the cross-over. Most of the methods currently used are based on the statistical probability of a root intersecting a fixed grid (eg Newman, 1966). Such methods are very time-consuming and prone to error. The imageanalysis based system described here offers a rapid and accurate method of determining root length and hence characterising the basic structural morphology of a plant root system.

The system consists of a Magiscan Image Analysis System with Genias 25 software (Joyce Loebl Ltd) in conjunction with a personal computer (80286 microprocessor and above), light table and black and white CCD camera equipped with a macro lens.

Roots are first carefully removed from the growing media, with great care taken to maintain structural integrity. This usually involves the use of sodium hexametaphosphate, a soil dispersant. Roots are then separated into orders $\left(1^{\circ}\right.$, $\left.2^{\circ} \mathrm{etc}\right)$ and the origin of each individual root noted, placed onto the light table in a suitable container, such as a Petri dish, and viewed with the camera. Roots $<0.1 \mathrm{~mm}$ can be resolved with the system and staining is not usually necessary. The video image is displayed on a monitor and a programme sequence which includes calibration automatically carried out. If necessary to improve contrast the normally linear relationship between the black and white video image and the grey image displayed on the monitor can be appropriately transformed. The system then allo-

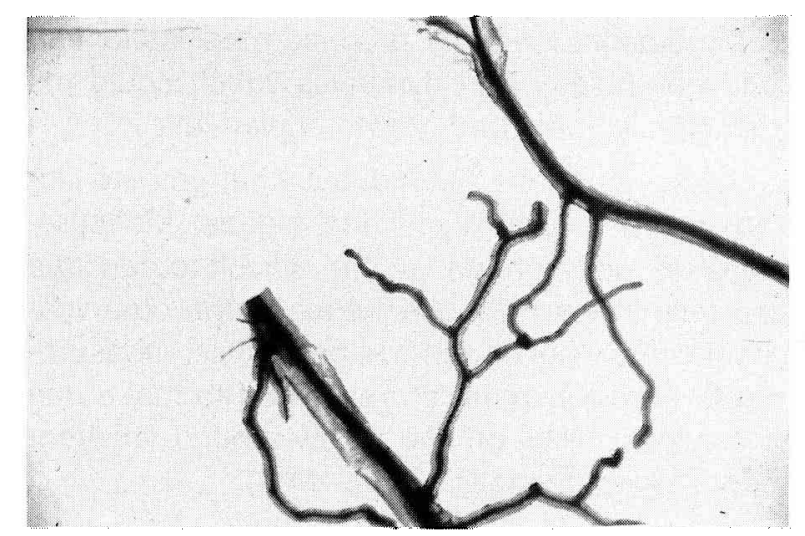

cates suitable threshold values ie minimum and maximum grey values and the grey image of the roots is converted into a binary image made up of individual elements, pixels, upon which future operations are performed. Pixels are then removed from the edges of the image until all lines are only 1 pixel thick - a skeletonised image of the roots is thus generated and the length of each sample subsequently measured by summing pixels. Underestimation of root length due to crossovers is minimised as all crossovers are only 1 pixel thick. Detailed characterisation of the root system is thus possible.

The value of the system can be shown in relation to measurements of the effects of AM colonisation or nutrients on the morphology of poplar root systems. Visual inspection of $2^{\circ}$ roots under a stereomicroscope suggests that AM colonised roots (fig 1a) are more branched than uncolonised roots (fig $1 \mathrm{~b}$ ). Image analysis performed as indicated above confirms this and shows that a higher percentage of the root systems of AM colonised plants is present as higher order laterals (fig 2).

\section{ROOT DYNAMICS}

In order to properly study root dynamics it is necessary to quantify a) root longevity, b) root fate, c) the periodicity of root growth and hence to observe roots non-destructively in situ over a period of time and to produce quantitative measurements based on these observations. The system described here represents a major advance in methodology. It consists of a miniature colour CCD television camera and video used in association with minirhizotron tubes for observation and a database and appropriate software for subsequent quantification and comparison of images over time.

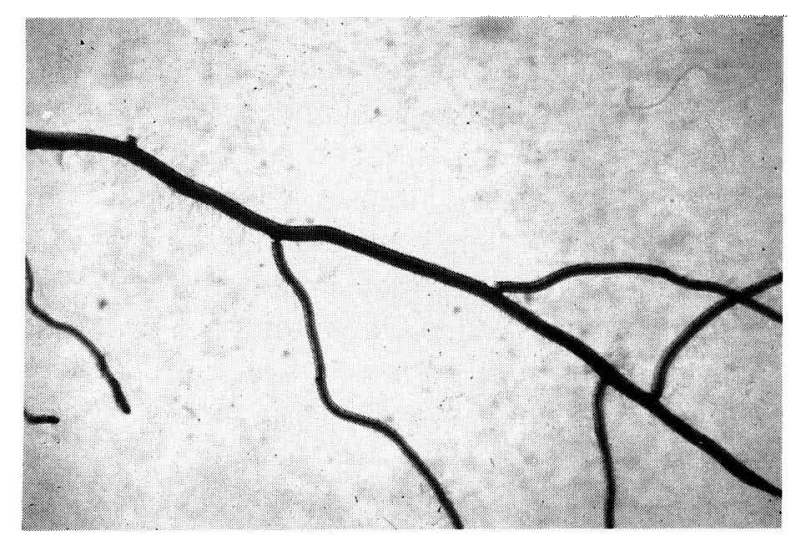

Fig 1. Morphology of roots (a) colonised and (b) not colonised by AM fungi. 


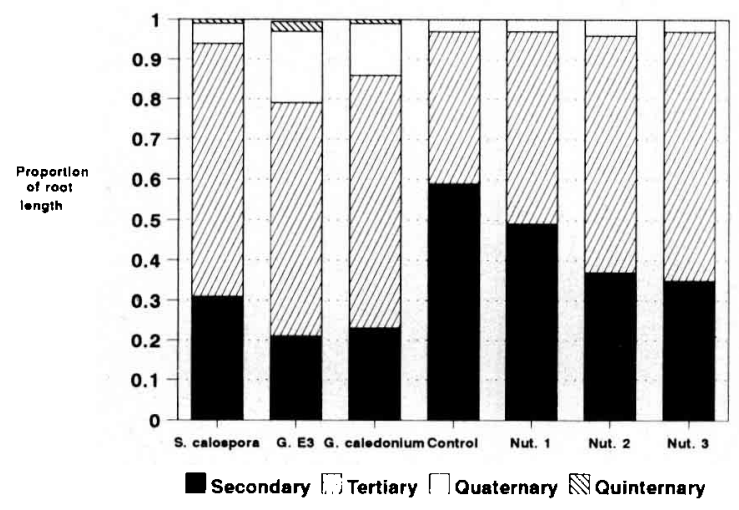

Fig 2. Effects of colonisation by $A M$ fungi or nutrient addition on the proportion of the root system of poplar made up of different order laterals (data from Hooker et al, 1992).

Minirhizotrons are essentially plastic or glass observation tubes inserted in the soil. These are then viewed with a miniature colour $C C D$ television camera with a $90^{\circ}$ viewing head (Bartz Technology Ltd). Excellent images of roots present at the tube/soil interface are obtained using this system and resolution of $<30 \mu \mathrm{m}$ is possible (fig 3 ). The camera and video system is fully portable and can be powered by either a 110,240 or $12 \mathrm{~V}$ source. To assess root longevity a cohort analysis approach is used, with the fate of individual roots produced in a given period being assessed. This requires that the same place on the observation tube be located at successive times. To do this fixed visual reference points are marked onto the minirhizotron tube. These are matched to corresponding marks on the monitor screen; thus it is possible to return to precisely the same positions over time and to record on video tape changes in individual roots occurring at the interface.

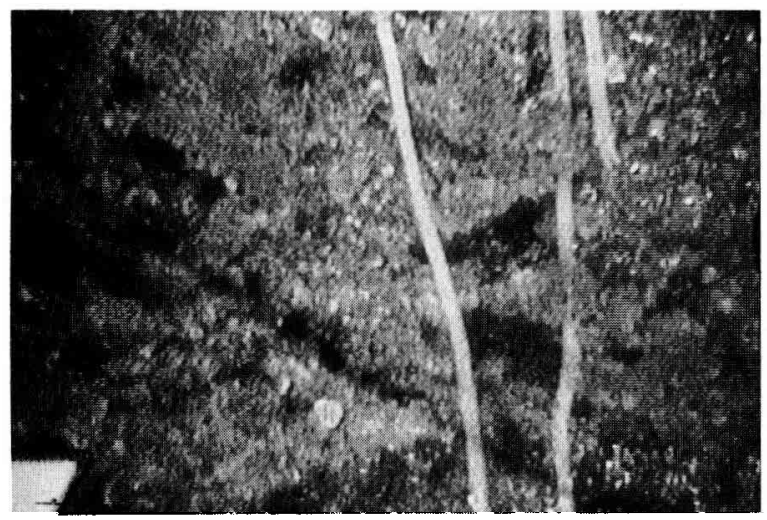

Fig 3. Image of roots obtained at the tube/soil interface using the minirhizotron camera system.
Images thus collected are compared over time. The image-analysis system uses an 80286 microprocessor-based personal computer equipped with a TARGA 16 image board (Truevision Inc) and CMAP Roots software (Hendrick and Pregitzer, 1992). Colour images are displayed on a secondary monitor and appropriate frames captured and displayed as a full colour digital image. Binary overlays of individual roots are then generated using a high-resolution optical mouse and labelled with an appropriate identifier; the length, area, condition and diameter may also be recorded (fig 4). This information is then added to a cumulative database ( $d$ Base III+, Ashton Tate) and comparison of individual records using software permits quantitative assessments of changes in root growth and condition over time. This approach has been used to compare the longevity of poplar roots (fig 5) where longevity of $<14 d$ is common.

\section{CONCLUSIONS}

The extent of modifications to plant root system morphology by AM fungi has only recently been appreciated and will have a major impact on our understanding of the mechanisms by which AM fungi influence plant uptake of water and the acquisition and cycling of nutrients in both natural and managed systems. There appears to be great potential to manipulate root system morphology to benefit plant establishment and growth in the ex vitro weaning and growth stages of micropropagation and other systems where AM fungi are not normally present.

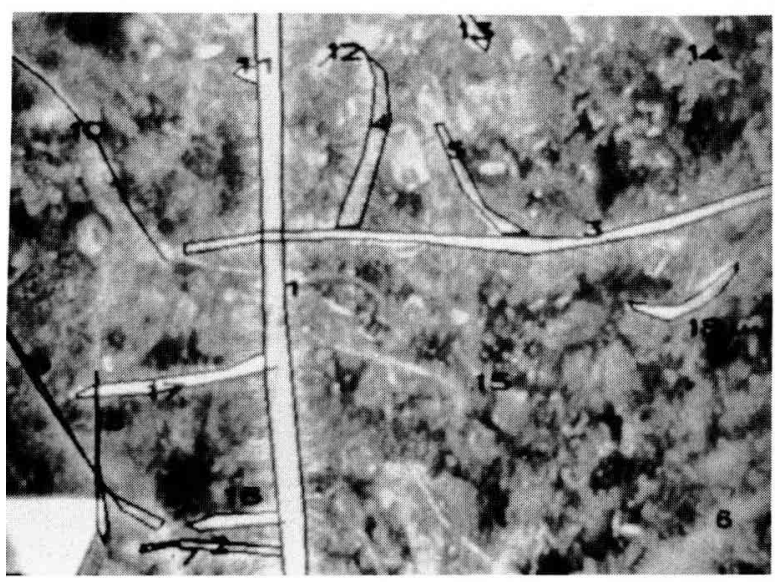

Fig 4. Binary overlays of roots occurring at the tube/soil interface; each root labelled with an identifier. 

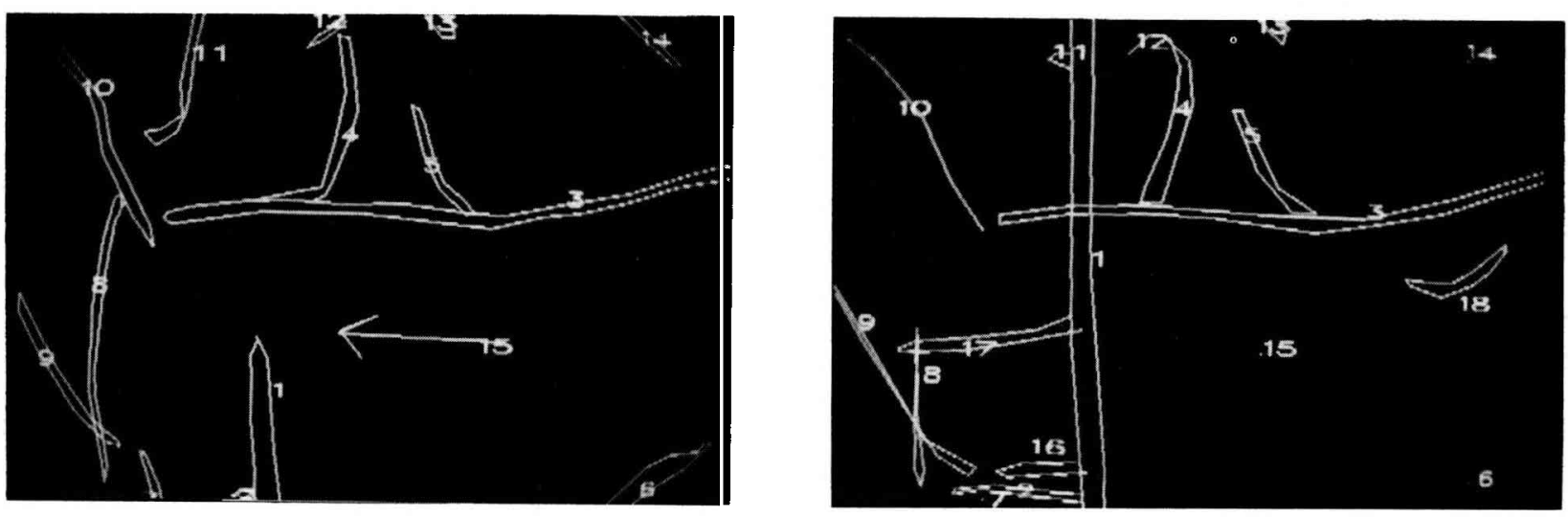

Fig 5. a. Binary overlay of poplar roots; b. $14 \mathrm{~d}$ later.

The 2 image-analysis systems described provide powerful tools to be used in the studies required to improve our understanding of the nature and extent of these modifications and the mechanisms involved.

\section{REFERENCES}

Atkinson (1989) Root growth and activity: current performance and future potential. Aspects Appl Biol 22, 1-14

Atkinson (1990) Influence of root system morphology and development on the need for fertiliser and the efficiency of use. In: Crops as Enhancers of Nutrient Use. Academic Press, NY, 411-451
Atkinson and Dawson (199I) Root growth: methods of measurement. In: Soil Analysis (Smith KA, Mullins CE, eds) Marcel Dekker, NY, 447-509

Berta G, Fusconi A, Trotta A and Scannerini S (1990) Morphogenetic modifications induced by the mycorrhizal fungus Glomus strain $E_{3}$ in the root system of Allium porrum L. New Phytol 114, 207-215

Hendrick RL and Pregitzer KS (1992) Spatial variation in tree root distribution and growth associated with minirhizotrons. Plant Soil 143, 283-288

Hooker JE et al (1992) Vesicular-arbuscular mycorrhizal fungi induced alteration in poplar root system morphology (in press)

Newman El (1966) A method of estimating the total length of root in a sample. $J$ App/ Ecol 3, 139-145 